\title{
Massive individual orbital angular momentum channels for multiplexing enabled by Dammann gratings
}

\author{
Ting Lei ${ }^{1, *}$, Meng Zhang ${ }^{2, *}$, Yuru Li ${ }^{3, *}$, Ping Jia ${ }^{2}$, Gordon Ning Liu ${ }^{4}$, Xiaogeng $\mathrm{Xu}^{4}$, Zhaohui Li ${ }^{3}$, Changjun Min ${ }^{1}$, \\ Jiao Lin ${ }^{5}$, Changyuan $\mathrm{Yu}^{6}$, Hanben $\mathrm{Niu}^{1}$ and Xiaocong Yuan ${ }^{1}$
}

Data transmission rates in optical communication systems are approaching the limits of conventional multiplexing methods. Orbital angular momentum (OAM) in optical vortex beams offers a new degree of freedom and the potential to increase the capacity of free-space optical communication systems, with OAM beams acting as information carriers for OAM division multiplexing (OAM-DM). We demonstrate independent collinear OAM channel generation, transmission and simultaneous detection using Dammann optical vortex gratings (DOVGs). We achieve 80/160 Tbit $^{-1}$ capacity with uniform power distributions along all channels, with 1600 individually modulated quadrature phase-shift keying (QPSK)/16-QAM data channels multiplexed by 10 OAM states, 80 wavelengths and two polarizations. DOVG-enabled OAM multiplexing technology removes the bottleneck of massive OAM state parallel detection and offers an opportunity to raise optical communication systems capacity to $\mathrm{Pbit} \mathrm{s}^{-1}$ level.

Light: Science \& Applications (2015) 4, e257; doi:10.1038/lsa.2015.30; published online 13 March 2015

Keywords: holographic gratings; multiplexing; optical communications; orbital angular momentum

\section{INTRODUCTION}

Optical communications in both free-space optical (FSO) and fiber optic technologies have achieved dramatic increments in their aggregate transmission rates using multiplexing/demultiplexing (MUX/ DEMUX) technologies. ${ }^{1}$ In addition to the multiplexing technologies based on amplitude, wavelength ${ }^{2-4}$ and light polarization ${ }^{5,6}$ division, the orbital angular momentum (OAM) of optical vortex (OV) beams ${ }^{7-11}$ provides a new degree of freedom for multiplexing to further increase the capacity of optical communications systems. OV beams have helical phase profiles in their electric fields that are proportional to the azimuthal phase term of $\exp (\mathrm{i} \ell \phi)$, where $\ell$ is the eigenvalue of the OAM or the so-called topological charge and $\phi$ is the azimuthal angle. In principle, the values of $\ell$ are unlimited, which makes it possible to use the OAM states as information channels to carry signals ${ }^{12-15}$ for data transmission. The superior security advantages of OAM channels have also been demonstrated in both classical and quantum communication systems. ${ }^{14-19}$

OAM-based optical communications can be classified into two different categories: OAM shift keying (OAM-SK) and OAM division multiplexing (OAM-DM). For OAM-SK, the OAM states of the OV beams are regarded as a modulation format that enables the encoding of information by dynamic switching of spatial light modulators. ${ }^{719-22}$
For OAM-DM, the OAM states are considered to be fixed channels to be used to carry information with the advantages of high bit rates and low bit error rates (BERs). ${ }^{13,23}$ In a state-of-the-art demonstration of

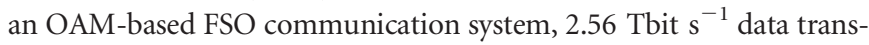
mission was obtained by the multiplexing of four OAM beams with two polarization states. ${ }^{13}$ Recently, the same research group achieved a data capacity of $100 \mathrm{Tbit} \mathrm{s}^{-1}$ through 1008 channels by multiplexing 42 wavelengths, 12 OAM beams and two polarization states together. ${ }^{24}$ However, in all the pioneering work described above, the maximum number of OAM channels that carried independent signals was only four, which is mainly constrained by the sophisticated experimental set-up required to detect multiple collinear OAM beams in parallel. Parallel detection of massive OAM channels is one of the major challenges in OAM-based optical communications. Another technique developed for OAM detection is known as the beam transformation approach. ${ }^{25,26}$ The donut-shaped OAM beam is transformed into a line shaped beam and can be focused onto spots distributed laterally depending on their topological charge. However, limited by the irregular shaped focal spots and small separation angles, this technique requires photodiode arrays to detect the separated OAM states, which is not compatible with the conventional optical communication system using single mode fiber collection. To enable further development of

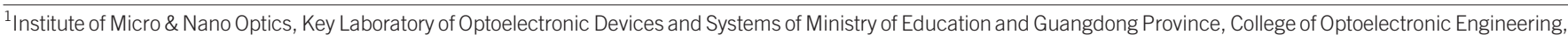

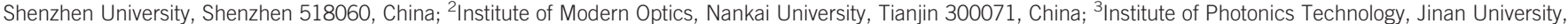

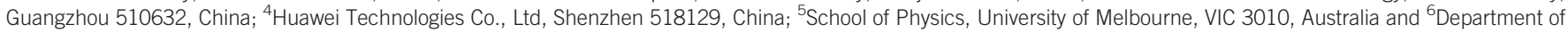
Electrical and Computer Engineering, National University of Singapore, 117576, Singapore

*These authors contributed equally to this work.

Correspondence: ZH Li, Institute of Photonics Technology, Jinan University, Guangzhou 510632, China

E-mail: tlzh88@jnu.edu.cn

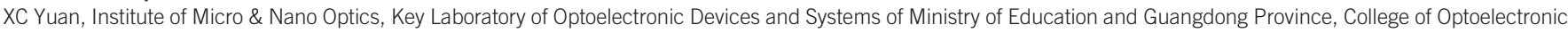
Engineering, Shenzhen University, Shenzhen 518060, China

E-mail: xcyuan@szu.edu.cn

Received 25 August 2014; revised 16 December 2014; accepted 18 December 2014; accepted article preview online 18 December 2014 
OAM-based optical communications, it will be necessary to introduce new approaches for fully independent data transmission and parallel detection using massive OAM channels combined in the MUX/ DEMUX technologies.

This article reports the design of a Dammann optical vortex grating (DOVG) for the multiplexing of massive OAM channels with individual modulation and simultaneous detection capabilities. In our previous work, ${ }^{27,28}$ we demonstrated the detection of OAM states in parallel using the DOVG, which provides uniform power distributions for all OAM states and relaxes the previously stringent beam alignment requirements. In the context of OAM-DM for high-speed optical communications, DOVGs can serve as key components to generate the massive OAM channels, multiplex these channels into coaxial OV beams and divide them equally for the detection process. OAMDM enabled by DOVG also has the ability to merge with standard wavelength division, polarization and other existing multiplexing technologies and achieve total data capacities of the sub-Pbit $s^{-1}\left(\sim 10^{14}\right.$ bit $\left.^{-1}\right)$ order.

\section{MATERIALS AND METHODS}

Figure 1 shows a schematic of the DOVG-enabled generation, multiplexing and demultiplexing of OAM for high-capacity FSO communications. Gaussian-shaped light beams with plane wavefronts $(\ell=0)$ carrying optical signals are incident on the DOVG at the angles of its diffraction orders, which are labeled as \pm 1 and \pm 2 in Figure 1a. Therefore, along the zeroth order of diffraction, all the incident Gaussian beams are encoded into different OAM channels in the combined coaxial OV beams. Figure $1 \mathrm{~b} 1$ shows the modeled wavefronts of the OAM with topological charges of $\ell= \pm 3$ and \pm 9 . Figure $1 \mathrm{~b} 2$ and $1 \mathrm{~b} 3$ shows the simulated intensity profiles and wavefronts of the coaxial OV beams. Because of the orthogonal property of OAM, the data carried by the coaxial OV beam can be transmitted in free space without crosstalk among the OAM channels. At the receiver end, all OAM channels are then demultiplexed into Gaussian beams at the corresponding diffraction orders by a second DOVG, which is identical to the grating used for multiplexing. Based on this DOVG-enabled OAM MUX/DEMUX scheme, high-capacity FSO communications can be attained by significantly increasing the number of OAM channels without the need for any additional bulk optics. The uniform power distributions across all the diffraction orders of the DOVGs indicate the high potential scalability of FSO systems with large numbers of high-order OAM channels (Supplementary Information).

We characterize several OAM beams $(\ell= \pm 3, \pm 9, \pm 15, \pm 21, \pm 27)$ that are generated, multiplexed and demultiplexed by our designed $1 \times 10$ DOVGs. Figure $1 \mathrm{~d} 1-1 \mathrm{~d} 5$ shows the intensity profiles of five of the OAM beams $(\ell=3,-9,15,-21,27)$ that were captured by an infrared camera at $1550 \mathrm{~nm}$ wavelength. These OAM beams have ringshaped profiles with low intensity at the center and radii proportional to $\sqrt{\ell}$, which is consistent with the theoretical results shown in Figure 1e1-1e5. Figure 1d6 shows an image of the combined coaxial OV beam with 10 OAM channels and, for comparison, Figure 1e6 shows the corresponding modeled beam pattern. Figure 1f and $1 \mathrm{~g}$ shows the measured and modeled intensity profiles of each OAM channel after demultiplexing, respectively. The ring-shaped beams are converted into bright high-intensity spots, which can then be coupled into optical fibers for detection.

Figure 2 shows a schematic diagram of the experimental set-up. The input signals are from a wavelength division multiplexing (WDM) system with 80 wavelengths, spanning from $1529.16 \mathrm{~nm}$ to $1560.61 \mathrm{~nm}$ with $50 \mathrm{GHz}$ spacing. At each wavelength, the light source is modulated to carry quadrature phase-shift keying (QPSK) signals

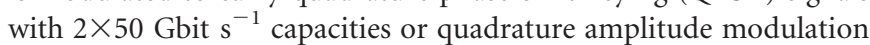
(16-QAM) signals with $2 \times 100 \mathrm{Gbit} \mathrm{s}^{-1}$ capacities, including the multiplexing of the $x$ - and $y$-polarizations (Supplementary Information). The output from the WDM system is divided equally into 10 branches, which match the diffraction orders of our designed 10-order DOVG. The signals from the 10 branches can be considered to be incoherent and unrelated after propagation in fibers for more than $10 \mathrm{~m}$. These signals are coupled from the fibers to free space with planar phase fronts through fiber collimators. When the beams pass through the DOVG at different angles, the diffraction beams in the zeroth order are loaded by the different OAM channels. We placed a pinhole aperture after the DOVG to filter out the higher orders of the diffracted beams
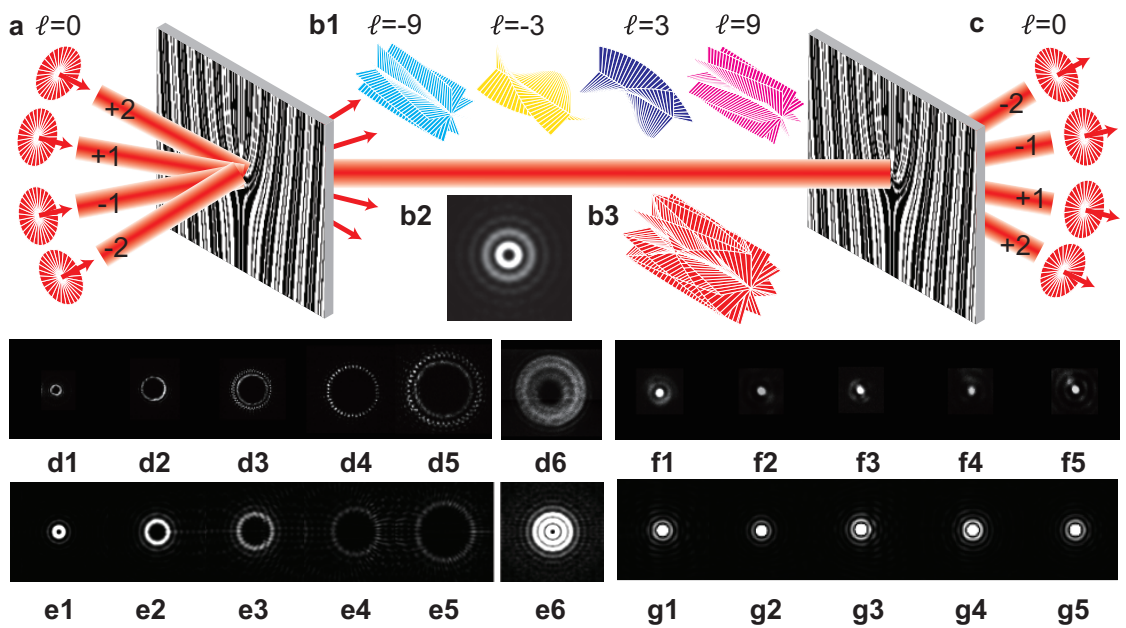

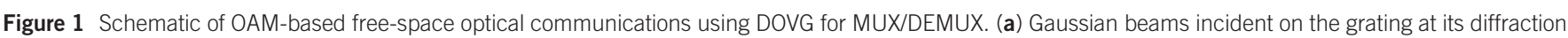

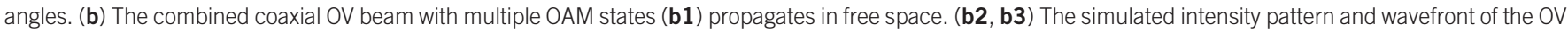

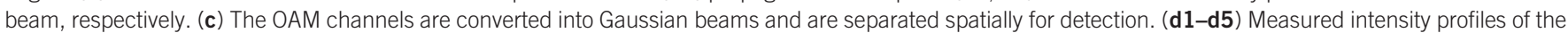

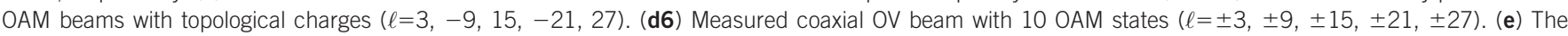

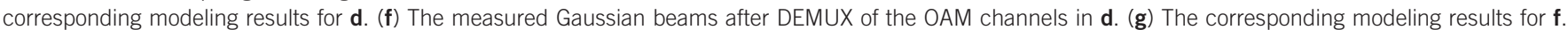
DOVG, Dammann optical vortex grating; MUX/DEMUX, multiplexing/demultiplexing; OAM, orbital angular momentum; OV, optical vortex. 


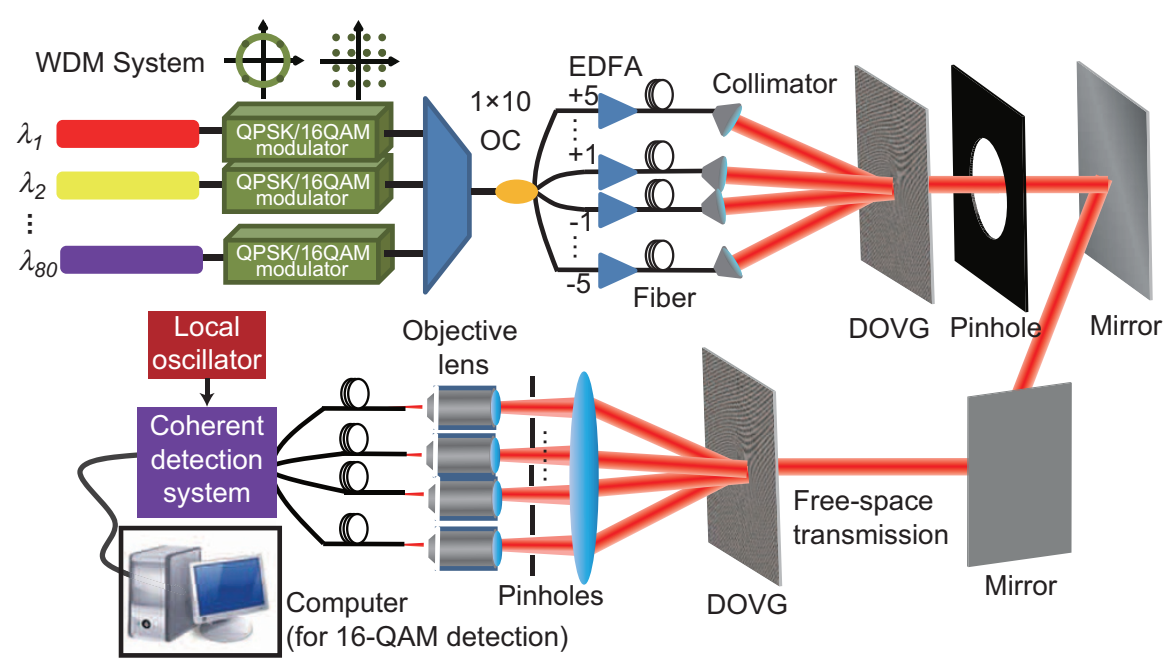

Figure 2 Experimental set-up of the OAM-based FSO system. A laser source with 80 wavelengths carrying QPSK/16-QAM signals and two polarizations is divided into 10 branches. Each branch is amplified by an EDFA and coupled into one of the OAM channels in the coaxial OV beams via $1 \times 10$ DOVG. After propagation in free space for $1 \mathrm{~m}$, the OAM channels are then diffracted into the corresponding angles and converted into Gaussian beams by an identical DOVG. The focused Gaussian beams pass through pinholes and are coupled into single mode fibers. The wavelength, polarization and QPSK/6-QAM signals are demultiplexed and all the encoded signals are detected in parallel. DOVG, Dammann optical vortex grating; EDFA erbium-doped fiber amplifier; FSO, free-space optical; OAM, orbital angular momentum; OV, optical vortex; 16-QAM, 16-quadrature amplitude modulation; QPSK, quadrature phase-shift keying ;WDM, wavelength division multiplexing; OC, optical coupler.
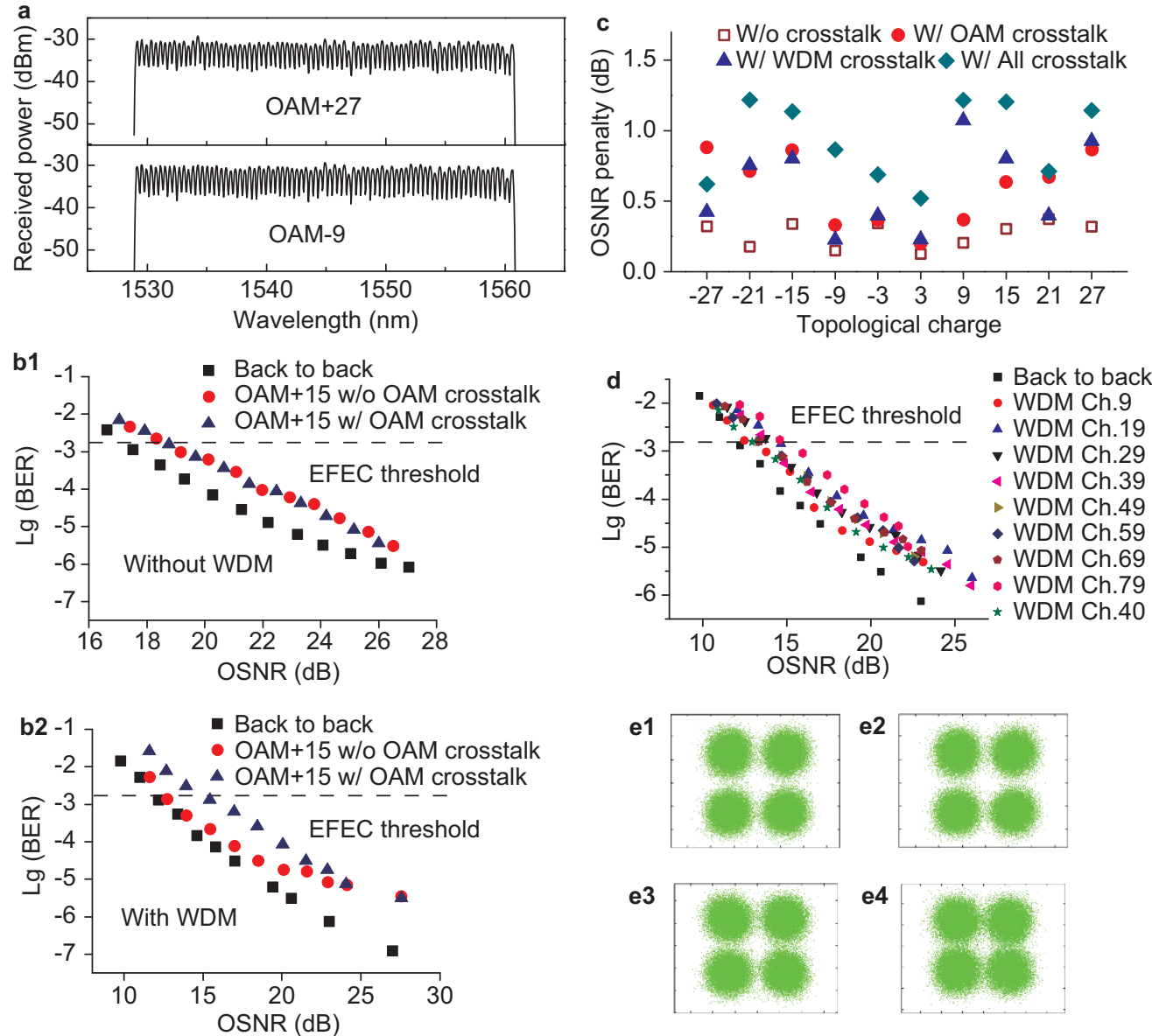

e1

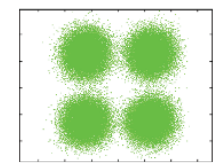

e3

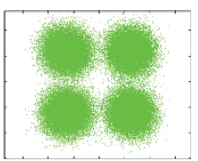

e2

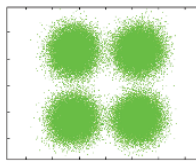

e4

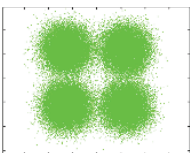

Figure 3 Experimental results for OAM-based FSO communications using QPSK signals. (a) Spectra of the OAM states +27 and -9 with the 80-wavelength WDM system. (b) Measured BERs of the OAM channels without (b1) and with (b2) the WDM system. (c) OSNR penalties of the 10 OAM states. (d) BER characteristics in the same OAM channel $\left(\ell=-15\right.$ ) for the 10 different wavelength channels. (e) Constellations of the QPSK signals: with BER of $5 \times 10^{-4}$ in OAM channels $\ell=3$ (e1) and $\ell=-21$ (e2); with BERs of $1.2 \times 10^{-3}(\mathrm{e} 3)$ and $3.3 \times 10^{-3}$ (e4) in OAM channel $\ell=3$. BER, bit error rate; FSO, free-space optical; OAM, orbital angular momentum; QPSK, quadrature phase-shift keying; WDM, wavelength division multiplexing; OSNR, optical signal-to-noise ratio; EFEC, enhanced forward error correction; w/, with; w/o, without. 
and ensure that only the zeroth order beams can pass through. After propagation in free space over a distance of $1 \mathrm{~m}$, the OV beams with the multiplexed OAM channels are demultiplexed using another grating with exactly the same parameters. When passing through the demultiplexing grating, the coaxial OAM beams are diffracted into 10 orders. The signal beam with a topological charge induced at a certain diffraction angle will be compensated and converted into a Gaussian beam with a bright high-intensity spot at its center. Beams with other topological charges will have ring shapes with low intensity at their centers. We use additional pinhole apertures to filter out these rings. The focused Gaussian beam is then coupled into a fiber by a microscopic objective and is analyzed using a real-time coherent detection system to measure the BER. The optical signal-to-noise ratio (OSNR) is measured using a spectrometer.

\section{RESULTS AND DISCUSSION}

We achieve a data capacity of 80 Tbit $\mathrm{s}^{-1}$ by multiplexing 1600 channels of $50 \mathrm{Gbit} \mathrm{s}^{-1}$ QPSK signals at 80 wavelengths with $10 \mathrm{OAM}$ states and two polarizations. It should be noted that in this system, all channels can be individually modulated, detected and simultaneously analyzed in real time.

Figure 3a shows the measured optical spectra for OAM states +27 and -9 at the receiver end. The 80 peaks in each spectrum are the 80 wavelengths multiplexed together. From these spectra, we can calibrate the insertion losses (from the transmitter end to the receiver end) for all 10 OAM channels in the range from $33.22 \mathrm{~dB}$ to $34.03 \mathrm{~dB}$, including $\sim 10 \mathrm{~dB}$ loss per DOVG, $\sim 0.5 \mathrm{~dB}$ loss per lens and $\sim 10 \mathrm{~dB}$ loss for the processes of passing through the pinholes and coupling into the single mode fiber. The diffraction losses of the DOVG for the 10 orders are in the 9.5-11.3 dB range. Therefore, we can claim that DOVGs enable relatively uniform power distributions for all OAM channels and relax the power constraints used for high-order OAM multiplexing in conventional diffraction gratings. We also measured the BERs of the transmitted signals for all OAM states under different conditions. Figure 3b1 shows the measured BER curves, which depend on the OSNR, at a wavelength of $1544.3 \mathrm{~nm}$ without the WDM system. Because the QPSK modulator/coherent detection system is packaged with polarization multiplexing/demultiplexing, each measured BER is the average value of the $x$ - and $y$-polarizations. The various OSNR values shown in the measurements are attained by controlling the power of the induced white noise. The data points labeled using black squares are the BERs for the back to back measurements, which means that the data transmission occurs directly from the modulator to the detector without the free space propagation. The data points labeled using red circles are the BERs of the signals transmitted from an OAM channel $(\ell=+15)$ with all other OAM channels switched off. Therefore, in this case, there is no crosstalk between the different OAM channels. The data labeled using blue triangles are the BERs of the signal from the same OAM channel but with all other OAM channels switched on. We see that the data transmissions with all OAM channels available have the highest BER values and gradually approach the values of the case without the OAM crosstalk with increasing OSNR. We attribute this behavior to the superior orthogonal properties of the OAM channels in FSO. With a suitable OSNR, the crosstalk among the different OAM channels can be suppressed. We repeat the BER measurements using an 80-wavelength WDM system as the input light source. Figure $3 \mathrm{~b} 2$ shows the BER-OSNR curves for $\ell=15$ at $\lambda=1544.53 \mathrm{~nm}$. We set the enhanced forward error correction (EFEC) threshold as a BER of $2 \times 10^{-3}$ and calculated the OSNR penalties for OAM-DM. Without the OAM crosstalk, the penalties for all
OAM channels are less than $0.5 \mathrm{~dB}$. Even with the OAM crosstalk, the penalties are still less than $1.5 \mathrm{~dB}$. To further characterize the performance of the WDM/OAM-DM system, we also compare the BERs in the same OAM channel $(\ell=-15)$ at different wavelengths, as shown in Figure $3 \mathrm{~d}$. For visualization purposes, we also measured the constellations of the QPSK signals from the demultiplexed OAM beams. Figure $3 \mathrm{e} 1$ and $3 \mathrm{e} 2$ shows the constellations of the OAM channels $\ell=3$ and $\ell=-21$ at a BER of $5 \times 10^{-4}$. The similarity of the two figures proves that the performances of the different OAM channels are similar. Figure 3e 3 and $3 \mathrm{e} 4$ shows the constellations at BERs of $1.2 \times 10^{-3}$ and $3.3 \times 10^{-3}$ of OAM channel $\ell=3$. We can see that an overlap appears in the constellation when the BER exceeds the EFEC threshold $\left(2 \times 10^{-3}\right)$, which means that the bit error cannot be corrected.

We have demonstrated the OAM-based free-space data link of $1 \mathrm{~m}$ length in the proof of concept experiment. To estimate the performance of OAM-based MUX/DEMUX technology for long-distance FSO, we model the OAM beams' propagation in an atmosphere based on the Kolmogorov turbulence theory. ${ }^{29-33}$ We take the random intensity and phase perturbation of OAM beams into account and calculate the crosstalk among the OAM states. We define $S_{m n}$ as the percentage of beam energy transferred from the OAM state $m$ to $n$. While $m=n, S_{m n}$ is the power remaining in the original OAM state $m$; while $m \neq n, S_{m n}$ is the crosstalk between OAM states $m$ and $n$. We define $C_{n}^{2}$ as the refractive-index structure parameter with a unit of $m^{-2 / 3}$, which is related to the strength of turbulence in the atmosphere.

For $\ell=3$ as the input OAM state (labeled as $\Delta \ell=0$ ), we model the $S_{m n}$ parameters of the input OAM state and the nearby states with topological charge differences $\Delta \ell$ from 1 to 6 after 1, 100, 500 and $1000 \mathrm{~m}$ propagation in some weak $\left(C_{n}^{2}=10^{-17} \mathrm{~m}^{-2 / 3}\right)$ and moderate $\left(C_{n}^{2}=10^{-16} \mathrm{~m}^{-2 / 3}\right)$ turbulence. Figure 4 a shows that there is still $90 \%$ energy remaining in the input OAM state after the $1000 \mathrm{~m}$ propagation in the atmosphere with the weak turbulence. We also find from the curves that a greater topological charge difference $\Delta \ell$ gives a smaller energy coupling $S_{m n}$ between the OAM states. For the two adjacent OAM communication channels $\ell=3$ and $\ell=9$ in our experiment, labeled as $\Delta \ell=0$ and $\Delta \ell=6$, respectively, the crosstalk is less than $0.2 \%$. According to the modeling results, we claim that the demonstrated OAM based FSO system is robust enough to overcome the crosstalk induced by kilometer-length propagation in the weak turbulence. By contrast, Figure $4 \mathrm{~b}$ shows that there is only approximately $60 \%$ energy remaining in the input OAM state after the $1000 \mathrm{~m}$ propagation in the atmosphere with moderate turbulence. In this case, the crosstalk between the input and adjacent OAM channels is above the EFEC threshold and the error bits cannot be corrected. Therefore, under the moderate and strong turbulence conditions, we need to adopt some adaptive optics approaches ${ }^{34}$ to compensate for the fluctuations of OAM-based long-distance free-space communication.

The DOVG with its pure phase structures is described using

$$
\Psi=\exp (\mathrm{i} \Phi)=\sum_{n=-\frac{N}{2}}^{\frac{N}{2}} E_{n} \exp \left[i n \times\left(\frac{2 \pi x}{T}+l \theta\right)\right]
$$

where $\Phi$ is the phase function, $N$ is the total number of diffraction orders (which is an even positive integer), $T$ is the period of the grating, $n$ is the diffraction order from $-N / 2$ to $N / 2, \theta$ is the azimuth angle in polar coordinates and $l$ is the interval of the topological charges, which is a non-zero integer. $\left|E_{n}\right|^{2}=1 / N$ is the power of the $n$th order normalized with respect to the total power. According to the above equation, a beam with a plane wavefront incident on the DOVG is diffracted into $N$ orders, each with equal energy carrying topological 

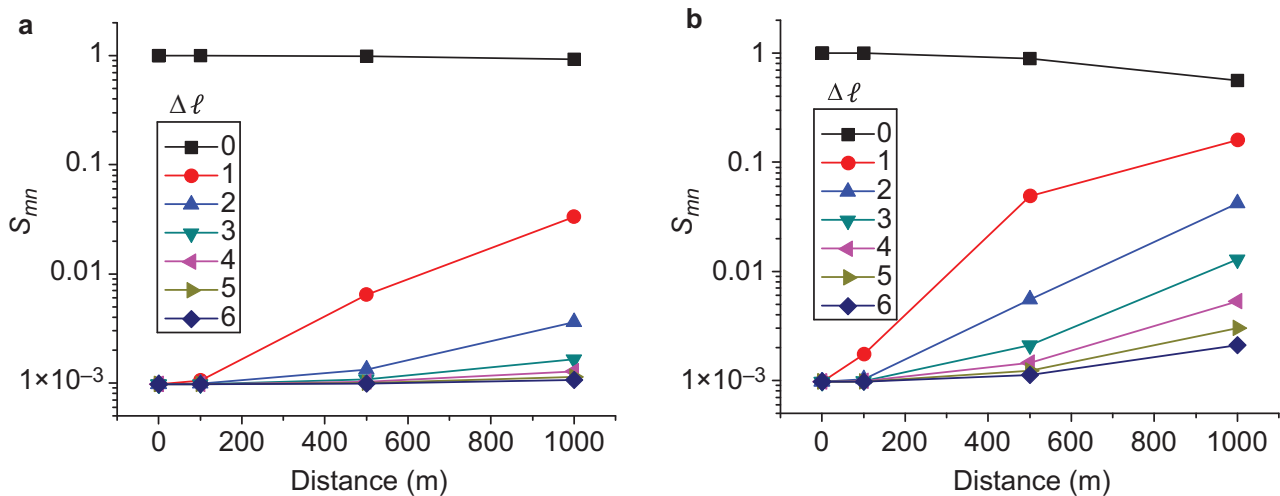

Figure 4 The $S_{m n}$ parameters of the input OAM state $(\ell=3$, labeled as $\Delta \ell=0)$ and the neighborhood states upon the propagation in the atmosphere with weak and moderate turbulence. (a) The energy percentage of the input OAM state and the nearby states after propagation for $1,100,500$ and $1000 \mathrm{~m}$ in a weak turbulence atmosphere. (b) The energy percentage of the input OAM state and the nearby states after propagation for 1, 100, 500 and $1000 \mathrm{~m}$ in a moderate turbulence atmosphere. $\Delta \ell$ is the topological charge difference between the measured state and the input state. OAM, orbital angular momentum.

charge $n \times l$. As an example, Figure 5a shows the modeling results for OAM beams $(\ell=+3,-3,+6$ and -6$)$ generated from a Gaussian beam passing through a $1 \times 4$ DOVG.

When we implement the DOVG in an inverse manner, multiple OAM beams will be generated and will then be multiplexed into a coaxial OV beam. As shown in Figure 5b, for a beam with a plane wavefront incident from the $n$th order direction, $n=n_{1}(-N / 2$ $\leqslant n_{1} \leqslant N / 2$ ), the diffraction component in the zeroth order diffraction direction will be

$$
a_{n_{1}}=E_{n_{1}} \times \exp \left(i n_{1} l \theta\right)
$$

With the number of plane waves $N$ incident on the grating from the corresponding diffraction direction of the DOVG, the complex amplitude distribution of the collinear OV beam in the zeroth order direction is

$$
A=\sum_{n=-\frac{N}{2}}^{\frac{N}{2}} E_{n} \exp (\text { inl } \theta)
$$

Therefore, the multiple incident beams from different angles can synthesize a coaxial beam loaded with different OAM channels using a DOVG and a pinhole filter. OAM multiplexing also introduces an insertion loss of $\sim 10 \times \lg (N) \mathrm{dB}$ because the high diffraction orders are all filtered by the pinhole.

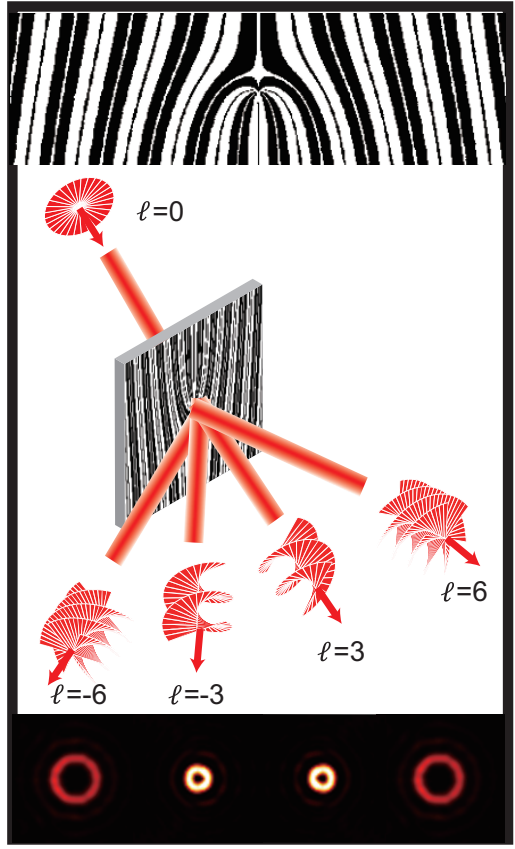

a

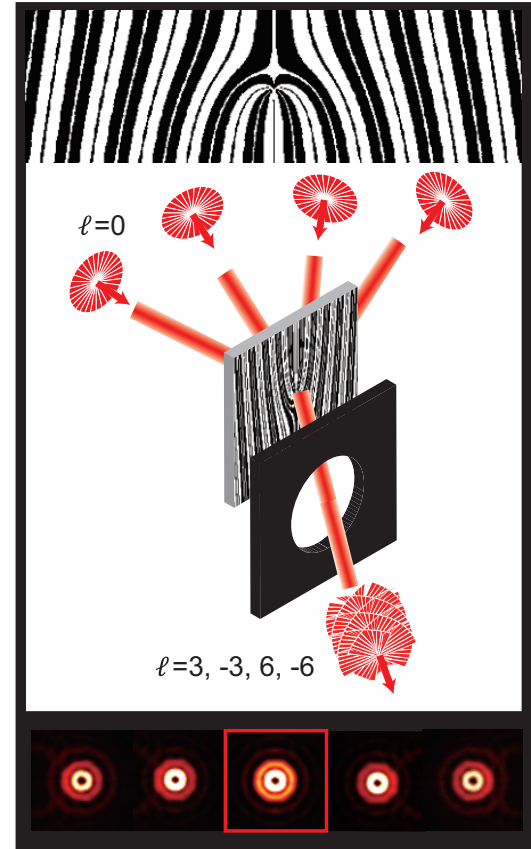

b

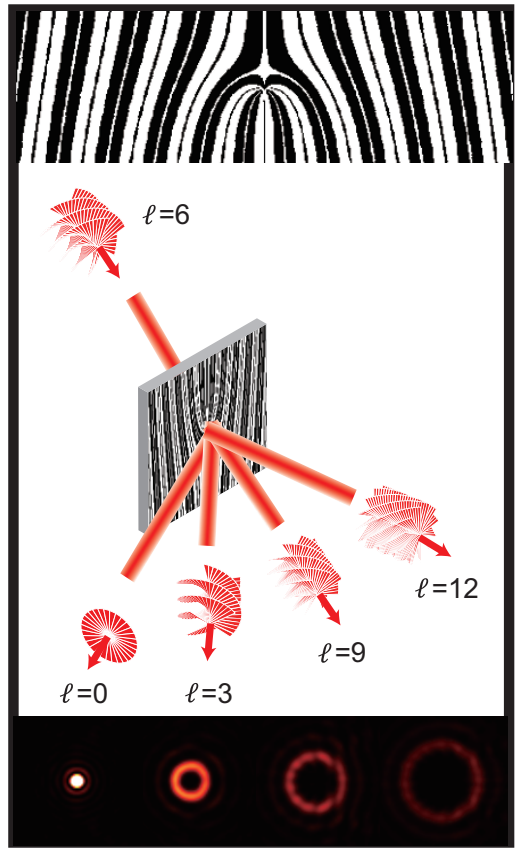

Figure 5 Principle of DOVG for OV beam combination and detection. (a) A Gaussian beam with a planar phase front is diffracted and encoded into OAM states by the DOVG. (b) Gaussian beams incident from the diffraction angles are combined into an OV beam with multiple OAM states in the zeroth order direction. (c) An OAM beam is diffracted and converted back into a Gaussian beam in the corresponding direction by an identical DOVG. DOVG, Dammann optical vortex grating; OAM, orbital angular momentum; OV, optical vortex. 
We use a DOVG with exactly the same parameters to demultiplex the OAM channels from the coaxial OV beam. When a beam with a specific topological charge (such as $n_{1} \times l$ ) is incident on the DOVG, the beam can be diffracted into $N$ orders, which can be described as

$$
A^{\prime}=\sum_{n=-\frac{N}{2}}^{\frac{N}{2}} E_{n} \exp \left[i n \times\left(\frac{2 \pi x}{T}+l \theta\right)+i n_{1} \times l \theta\right]
$$

The diffraction beam in the $-n_{1}$ th order (i.e., $n=-n_{1}$ ) is converted into a plane wave (with topological charge of 0 ), which can then be focused into a Gaussian-shaped light spot and coupled into an optical fiber for detection while the other diffraction orders are still ring spots, as shown in Figure $5 \mathrm{c}$. We must note that the diffraction energies are equally divided among all designed $N$ orders by the DOVG, which solves the problems of detection of weak signals from the higher orders of a conventional grating. Thus, the dynamic range of the OAM channel detection is improved by the DOVG. Incident coaxial OV beams carrying massive OAM channels can be demultiplexed in parallel by a single DOVG. There is no need for an additional optical splitter or for complex computer-generated holography in the detection system.

In the experiment, we used an optimized binary $(0-\pi)$ phase vortex Dammann grating for the OAM multiplexing/demultiplexing processes. This binary phase element has the advantages of simple manufacture and low cost. The DOVG not only simplifies the system complexity significantly, but also extends the OAM detection range and increases the system capacity. According to our simulation results, the grating can detect tens or even hundreds of OAM channels simultaneously.

\section{CONCLUSIONS}

Since OAM is a new multiplexing technology, it is natural that we question the level to which we can increase the channel numbers while maintaining reasonable performance for practical communication applications. In this article, we report the DOVG-enabled parallel detection of independent QPSK/16-QAM signals from 10 OAM channels as an example to prove our concept. We choose topological charge values of OAM with a relatively large interval of 6 to reduce interference between the collinear OAM channels, where a large OAM dynamic range is demonstrated experimentally. Even with an OAM interval of 1 , the measured BERs are still above the EFEC threshold in our communication system. For one-dimensional DOVG, we estimate that several tens of OAM channels can be properly detected with sufficient diffraction angle intervals to arrange the appropriate filters and detectors. If we extend the DOVG to produce a two-dimensional element, the spatial constraints for massive OAM channels detection will be greatly relaxed. For example, we can increase the detection range of the OAM states from \pm 2 to \pm 12 with the same diffraction angles by replacing the one-dimensional $(1 \times 5)$ DOVG with a two-dimensional $(5 \times 5)$ element. ${ }^{35}$ Another advantage of this method is immunity to stringent alignment and phase matching requirements between the light beams and the optical elements. ${ }^{7}$ This property can be used to simplify the set-up and improve its reliability for FSO communication systems. We therefore believe that the data capacity of FSO communications based on DOVG-enabled OAM-DM can be increased greatly.

\section{AUTHOR CONTRIBUTIONS}

TL, MZ and YL contributed equally to this paper. XY and ZL initiated and directed the project and oversaw the manuscript. TL, MZ, PJ, YL, GNL and XX were responsible for the experimental investigations. CM and JL were responsible for the theoretical studies. CY and HN were responsible for optical and communication systems.

\section{CONFLICTS OF INTEREST}

The authors declare no competing financial interests. Correspondence and requests for materials should be addressed to XY and ZL.

\section{ACKNOWLEDGEMENTS}

This work was partially supported by the National Natural Science Foundation of China under Grant numbers 61036013, 61138003, 61427819, 61001101 and 61435006. XY acknowledges support from the Ministry of Science and Technology of China under National Basic Research Program of China (973) grant no. 2015CB352004.

1 Essiambre R, Tkach RW. Capacity trends and limits of optical communication networks. Proc IEEE 2012; 100: 1035-1055.

2 Gnauck AH, Winzer PJ, Chandrasekhar S, Liu X, Zhu B et al. Spectrally efficient longhaul WDM transmission using 224-Gb/s polarization-multiplexed 16-QAM J Lightwave Technol 2011; 29: 373-377.

3 Zhou X, Yu JJ, Huang MF, Shao Y, Wang T et al. 64-Tb/s, 8 b/s/Hz, PDM-36QAM transmission over $320 \mathrm{~km}$ using both pre- and post-transmission digital signal processing. J Lightwave Technol 2011; 29: 571-577.

4 Sano A, Masuda H, Kobayashi T, Fujiwara M, Horikoshi K et al. Ultra-high capacity WDM transmission using spectrally-efficient PDM 16-QAM modulation and C- and extended L-band wideband optical amplification. J Lightwave Technol 2011; 29: 578-586.

5 Liu X, Chandrasekhar S, Chen X, Winzer PJ, Pan Y et al. 1.12-Tb/s 32-QAM-OFDM superchannel with $8.6-\mathrm{b} / \mathrm{s} / \mathrm{Hz}$ intrachannel spectral efficiency and space-division multiplexed transmission with $60-\mathrm{b} / \mathrm{s} / \mathrm{Hz}$ aggregate spectral efficiency. Opt Express 2011; 19: B958-B964.

6 Ryf R, Randel S, Gnauck AH, Bolle C, Sierra A et al. Mode-division multiplexing over $96 \mathrm{~km}$ of few-mode fiber using coherent $6 \times 6 \mathrm{MIMO}$ processing. J Lightwave Technol 2012; 30: 521-531.

7 Gibson G, Courtial J, Padgett M, Vasnetsov M, Pas'ko V et al. Free-space information transfer using light beams carrying orbital angular momentum. Opt Express 2004; 12 : 5448-5456.

8 Shapiro J, Guha S, Erkmen B. Ultimate channel capacity of free-space optical communications. J Opt Network 2005; 4: 501-516.

9 Djordjevic IB. Deep-space and near-Earth optical communications by coded orbital angular momentum (OAM) modulation. Opt Express 2011; 19: 14277-14289.

10 Awaji A, Wada, N, Toda, Y. Demonstration of spatial mode division multiplexing using Laguerre-Gaussian mode beam in telecom-wavelength. In: Proceedings of the IEEE Photonics Conference; 7-11 November 2010; Denver, CO, USA. IEEE: New York, USA, 2010, pp551-552.

11 Wang J, Yang JY, Fazal IM, Ahmed N, Yan Y et al. Experimental demonstration of 100 Gbit/s DQPSK data exchange between orbital-angular-momentum modes. In: Proceedings of the Optical Fiber Communication Conference; 4-8 March 2012; Los Angeles, CA, USA. Optical Society of America: Washington, DC, USA, 2012, pp665667.

12 Martelli P, Gatto A, Boffi P, Martinelli M. Free-space optical transmission with orbital angular momentum division multiplexing. Electron Lett 2011; 47: 972-973.

13 Wang J, Yang JY, Fazal IM, Ahmed N, Yan Y et al. Terabit free-space data transmission employing orbital angular momentum multiplexing. Nat Photonics 2012; 6: 488496.

14 Yao E, Franke-Arnold S, Courtial J, Barnett S, Padgett M. Fourier relationship between angular position and optical orbital angular momentum. Opt Express 2006; 14: 9071-9076.

15 Čelechovský R, Bouchal Z. Optical implementation of the vortex information channel. New J Phys 2007; 9: 328.

16 Jack B, Aursand P, Franke-Arnold S, Ireland DG, Leach J et al. Demonstration of the angular uncertainty principle for single photons. J Opt 2011; 13: 064017.

17 Leach J, Jack B, Romero J, Jha AK, Yao AM et al. Quantum correlations in optical angle-orbital angular momentum variables. Science 2010; 329: 662-665.

18 Franke-Arnold S, Barnett SM, Yao E, Leach J, Courtial J et al. Uncertainty principle for angular position and angular momentum. New J Phys 2004; 6: 103.

19 Gruneisen MT, Miller WA, Dymale RC, Sweiti AM. Holographic generation of complex fields with spatial light modulators: application to quantum key distribution. Appl Opt 2008; 47: A32-A42.

20 Jia P, Yang Y, Min CJ, Fang H, Yuan XC. Sidelobe-modulated optical vortices for freespace communication. Opt Lett 2013; 38: 588-590.

21 Gibson G, Courtial J, Vasnetsov M, Barnett S, Franke-Arnold S et al. Increasing the data density of free-space optical communications using orbital angular momentum. Proc SPIE 2004; 5550: 367-373.

22 Chagnon M, Osman M, Zhuge Q, Xu X, Plant DV. Analysis and experimental demonstration of novel 8PolSK-QPSK modulation at 5 bits/symbol for passive mitigation of nonlinear impairments. Opt Express 2013; 21: 30204-30220.

23 Bozinovic N, Yue Y, Ren YX, Tur M, Kristensen P et al. Terabit-scale orbital angular momentum mode division multiplexing in fibers. Science 2013; 340: 1545-1548. 
24 Huang $\mathrm{H}$, Xie GD, Yan Y, Ahmed N, Ren YX et al. 100 Tbit/s free-space data link enabled by three-dimensional multiplexing of orbital angular momentum, polarization, and wavelength. Opt Lett 2014; 39: 197-200.

25 Mirhosseini M, Malik M, Shi Z, Boyd RW. Efficient separation of the orbital angular momentum eigenstates of light. Nat Commun 2013; 4: 2781.

26 Berkhout G, Lavery M, Courtial J, Beijersbergen M, Padgett M. Efficient sorting of orbital angular momentum states of light. Phys Rev Lett 2010; 105: 153601.

27 Zhang N, Yuan XC, Burge RE. Extending the detection range of optical vortices by Dammann vortex gratings. Opt Lett 2010; 35: 3495-3497.

28 Wang ZX, Zhang N, Yuan XC. High-volume optical vortex multiplexing and demultiplexing for free-space optical communication. Opt Express 2011; 19: 482-492.

29 Andrews LC, Phillips RL, Hopen CY, Al-Habash MA. Theory of optical scintillation. JOSA A 1999; 16: 1417-1429.

30 Anguita JA, Neifeld MA, Vasic BV. Turbulence-induced channel crosstalk in an orbital angular momentum-multiplexed free-space optical link. Appl Opt 2008; 47: 2414 2429.

31 Rodenburg B, Lavery MPJ, Malik M, O'Sullivan MN, Mirhosseini M et al. Influence of atmospheric turbulence on states of light carrying orbital angular momentum. Opt Lett 2012; 37: 3735-3737.
32 Paterson C. Atmospheric turbulence and orbital angular momentum of single photons for optical communication. Phys Rev Lett 2005; 94: 153901.

33 Andrews LC. An analytical model for the refractive index power spectrum and its application to optical scintillations in the atmosphere. J Mod Opt 1992; 39: 1849-1853.

34 Ren Y, Xie G, Huang H, Bao C, Yan Y et al. Adaptive optics compensation of multiple orbital angular momentum beams propagating through emulated atmospheric turbulence. Opt Lett 2014; 39: 2845-2848.

35 Moreno I, Davis JA, Cottrell DM, Zhang N, Yuan XC. Encoding generalized phase functions on Dammann gratings. Opt Lett 2010; 35: 1536-1538.

(c) (i) $\Theta$ This work is licensed under a Creative Commons Attribution-

c. party material in this article are included in the article's Creative Commons license, unless indicated otherwise in the credit line; if the material is not included under the Creative Commons license, users will need to obtain permission from the license holder to reproduce the material. To view a copy of this license, visit http://creativecommons.org/licenses/ by-nc-nd/3.0/

Supplementary information for this article can be found on the Light: Science \& Applications' website (http://www.nature.com/lsa/). 